KYUNGPOOK Math. J. 49(2009), 265-281

\title{
On Applications of Differential Subordination to Certain Sub- class of Multivalent Functions
}

Rasoul AghalarY*

Department of Mathematics, University of Urmia, Urmia, Iran

e-mail : raghalary@yahoo.com

ZHI-GANG WANG

School of Mathematics and Computing Science, Changsha University of Science and Technology, Changsha 410076, Hunan, People's Republic of China

e-mail : zhigwang@163.com

ABSTRACT. In the present paper, we introduce and investigate a new subclass of multivalent functions associated with the Cho-Kwon-Srivastava operator $\tau_{p}^{\lambda}(a, c)$. Such results as inclusion relationships, convolution properties and criteria for starlikeness are proved. Relevant connections of the results presented here with those obtained in earlier works are also pointed out.

\section{Introduction and preliminaries}

Let $\mathcal{A}(p)$ denote the class of functions $f$ normalized by

$$
f(z)=z^{p}+\sum_{k=1}^{\infty} a_{p+k} z^{p+k} \quad(p \in \mathbb{N}:=\{1,2,3, \cdots\})
$$

which are analytic and $p$-valent in the open unit disc

$$
\mathbb{U}:=\{z: z \in \mathbb{C} \text { and }|z|<1\} .
$$

For simplicity, we write

$$
\mathcal{A}(1)=: \mathcal{A} \text {. }
$$

For two functions $f$ and $g$, analytic in $\mathbb{U}$, we say that the function $f(z)$ is subordinate to $g(z)$ in $\mathbb{U}$, and write

$$
f(z) \prec g(z) \quad(z \in \mathbb{U})
$$

if there exists a Schwarz function $\omega(z)$, which is analytic in $\mathbb{U}$ with

$$
\omega(0)=0 \quad \text { and } \quad|\omega(z)|<1 \quad(z \in \mathbb{U})
$$

\footnotetext{
* Corresponding author.
}

Received 11 March 2008; revised 24 June 2008; accepted 3 June 2008.

2000 Mathematics Subject Classification: 30C45, 30C80.

Key words and phrases: Cho-Kwon-Srivastava operator, differential subordination, Hadamard product (or convolution), starlike and convex functions. 
such that

$$
f(z)=g(\omega(z)) \quad(z \in \mathbb{U}) .
$$

Indeed it is known that

$$
f(z) \prec g(z) \quad(z \in \mathbb{U}) \rightarrow f(0)=g(0) \quad \text { and } \quad f(\mathbb{U}) \subset g(\mathbb{U}) .
$$

Furthermore, if the function $g$ is univalent in $\mathbb{U}$, then we have the following equivalence:

$$
f(z) \prec g(z) \quad(z \in \mathbb{U}) \Longleftrightarrow f(0)=g(0) \quad \text { and } \quad f(\mathbb{U}) \subset g(\mathbb{U}) .
$$

A function $f \in \mathcal{A}(p)$ is said to be in the class $\mathcal{S}_{p}^{*}(\alpha)$, the space of $p$-valently starlike functions of order $\alpha, 0 \leq \alpha<p$, if and only if it satisfies the following inequality:

For $p=1$, we write

$$
\Re\left(\frac{z f^{\prime}(z)}{f(z)}\right)>\alpha \quad(z \in \mathbb{U})
$$

$$
\mathcal{S}_{1}^{*}(\alpha)=: \mathcal{S}^{*}(\alpha) .
$$

A function $f \in \mathcal{A}(p)$ is said to be in the class $\mathcal{K}_{p}(\alpha)$ of all $p$-valently convex functions of order $\alpha, 0 \leq \alpha<p$, if and only if

$$
\frac{z f^{\prime}}{p} \in \mathcal{S}_{p}^{*}(\alpha) .
$$

As usual, for $p=1, \mathcal{K}_{1}(0)=: \mathcal{K}$ denote the family of all convex functions in $\mathbb{U}$. Also a function $f \in \mathcal{A}$ is said to be strongly starlike of order $\beta, \beta>0$, if and only if it satisfies the following subordination condition:

$$
\frac{z f^{\prime}(z)}{f(z)} \prec\left(\frac{1+z}{1-z}\right)^{\beta} \quad(z \in \mathbb{U}),
$$

and is denoted by $\mathcal{S}(\beta)$.

For functions $f_{j}(z) \in \mathcal{A}(p)$, given by

$$
f_{j}(z)=z^{p}+\sum_{k=1}^{\infty} a_{p+k, j} z^{p+k} \quad(j=1,2),
$$

we define the Hadamard product (or convolution) of $f_{1}(z)$ and $f_{2}(z)$ by

$$
\left(f_{1} * f_{2}\right)(z):=z^{p}+\sum_{k=1}^{\infty} a_{p+k, 1} a_{p+k, 2} z^{p+k}=:\left(f_{2} * f_{1}\right)(z) .
$$

In terms of the Pochhamer symbol (or the shifted function) $(k)_{n}, k \in \mathbb{R}$, given by

$$
(k)_{n}:= \begin{cases}1, & (n=0) ; \\ k(k+1)(k+2) \cdots(k+n-1), & (n \in \mathbb{N}),\end{cases}
$$


we now define a function $\phi_{p}(a ; c ; z)$ by

$$
\begin{gathered}
\phi_{p}(a ; c ; z):=z^{p}+\sum_{k=1}^{\infty} \frac{(a)_{k}}{(c)_{k}} z^{p+k} \\
\left(a \in \mathbb{R} ; c \in \mathbb{R} \backslash \mathbb{Z}_{0}^{-} ; \mathbb{Z}_{0}^{-}:=\{0,-1,-2, \cdots\} ; z \in \mathbb{U}\right) .
\end{gathered}
$$

Corresponding to the function $\phi_{p}(a ; c ; z)$ given by (1.3), we consider a linear operator:

$$
\mathcal{L}_{p}(a, c) f(z): \mathcal{A}_{p} \rightarrow \mathcal{A}_{p}
$$

which is defined by means of the following Hadamard product (or convolution):

$$
\mathcal{L}_{p}(a, c) f(z):=\phi_{p}(a, c ; z) * f(z) \quad\left(f \in \mathcal{A}_{p}\right),
$$

It is easily seen from (1.3) and (1.4) that

$$
z\left(\mathcal{L}_{p}(a, c) f(z)\right)^{\prime}=a \mathcal{L}(a, c) f(z)-(a-p) \mathcal{L}(a, c) f(z),
$$

we also note that

$$
\mathcal{L}_{p}(a, a) f(z)=f(z), \quad \mathcal{L}_{p}(p+1, p) f(z)=\frac{z f^{\prime}(z)}{p}
$$

and

$$
\mathcal{L}_{p}(n+p, 1) f(z)=D^{n+p-1} f(z) \quad(n>-p),
$$

where, in the special case, when

$$
p=1 \quad \text { and } \quad n \in \mathbb{N}_{0} \quad\left(\mathbb{N}_{0}:=\mathbb{N} \cup\{0\}\right),
$$

$D^{n}$ can be identified with the Ruscheweyh derivative of order $n$, (see, for details, [6]). With the aid of the function $\phi_{p}(a ; c ; z)$ defined by (1.3), we here consider a function $\phi_{p}^{\lambda}(a, c ; z)$ given by the following convolution:

$$
\phi_{p}(a, c ; z) * \phi_{p}^{\lambda}(a, c ; z)=\frac{z^{p}}{(1-z)^{\lambda+p}} \quad(\lambda>-p),
$$

which leads us to the following family of linear operators:

$$
\begin{gathered}
\tau_{p}^{\lambda}(a, c) f(z):=\phi_{p}^{\lambda}(a, c ; z) * f(z) \\
\left(a, c \in \mathbb{R} \backslash \mathbb{Z}_{0}^{-} ; \lambda>-p ; z \in \mathbb{U} ; f \in \mathcal{A}_{p}\right) .
\end{gathered}
$$

It is readily verified from the definition (1.5) that

$$
z\left(\tau_{p}^{\lambda}(a+1, c) f(z)\right)^{\prime}=a \tau_{p}^{\lambda}(a, c) f(z)-(a-p) \tau_{p}^{\lambda}(a+1, c) f(z),
$$


and

$$
z\left(\tau_{p}^{\lambda}(a, c) f(z)\right)^{\prime}=(\lambda+p) \tau_{p}^{\lambda+1}(a, c) f(z)-\lambda \tau_{p}^{\lambda}(a, c) f(z) .
$$

Also by definition and specializing the parameters $\lambda, a, c$, we obtain

$$
\begin{gathered}
\tau_{p}^{1}(p+1,1) f(z)=f(z), \quad \tau_{p}^{1}(p, 1) f(z)=\frac{z f^{\prime}(z)}{p}, \\
\tau_{p}^{n}(a, a) f(z)=D^{n+p-1} f(z) \quad(n>-p),
\end{gathered}
$$

and

$$
\tau_{p}^{\mu}(\mu+p+1,1) f(z)=F_{\mu, p}(f)(z) \quad(\mu>-p),
$$

where $F_{\mu, p}$ denote a familiar integral operator defined by (2.4) below (see Section 2 ). In recent years, the operator $\tau_{p}^{\lambda}(a, c)$ was studied by many authors (see, for details, Cho et al. [2], Patel [4], Aghalary [1] and Sokółand Trojnar-Spelina [7]).

Making Use of the Cho-Kwon-Srivastava operator $\tau_{p}^{\lambda}(a, c)$, we now define a new subclass of $\mathcal{A}_{p}$ as follows.

Definition 1.1. For fixed parameters, $\mu>0$, and $\alpha \in \mathbb{C}-\{0\}$, with $\Re \alpha \geq 0$, we say that a function $f \in \mathcal{A}_{p}$ is in the class $R_{a, c}^{\lambda}(p, \alpha, \mu)$ if it satisfies the following subordination condition:

$$
(1-\alpha) \frac{\tau_{p}^{\lambda}(a, c) f(z)}{z^{p}}+\frac{\alpha}{p} \frac{z\left(\tau_{p}^{\lambda}(a, c) f(z)\right)^{\prime}}{z^{p}} \prec 1+\mu z .
$$

The object of the present paper is to obtain several inclusion relationships and other interesting properties of functions belonging to the subclass $R_{a, c}^{\lambda}(p, \alpha, \mu)$. By using the method of differential subordination, some mapping properties involving the Cho-Kwon-Srivastava operator $\tau_{p}^{\lambda}(a, c)$ are also investigated.

In order to prove our main results, we shall require the following lemmas.

Lemma 1.1(Hallenbeck and Rusheweyh [3]). Let $h(z)$ be analytic and convex univalent in the unit disk $\mathbb{U}$ with $h(0)=a, c \neq 0, \Re(c) \geq 0$. Also let

$$
g(z)=a+b_{n} z^{n}+b_{n+1} z^{n+1}+\cdots
$$

be analytic in $\mathbb{U}$. If

$$
g(z)+\frac{z g^{\prime}(z)}{c} \prec h(z) \quad(z \in \mathbb{U}),
$$

then

$$
g(z) \prec \psi(z)=\frac{c}{z^{\left(\frac{c}{n}\right)}} \int_{0}^{z} t^{\left(\frac{c}{n}\right)-1} h(t) d t \prec h(z) \quad(z \in \mathbb{U}) .
$$


and the function $\psi$ is convex and is the best dominant of (1.11).

Lemma 1.2(Ruscheweyh and Stankiewicz [5]). If $f, g$ are analytic and $F, G \in \mathcal{K}$ such that $f \prec F, g \prec G$, then $f * g \prec F * G$.

\section{Main results}

We begin by stating our first result given by Theorem 2.1 below.

Theorem 2.1. Let $a>0,0 \neq \alpha \in \mathbb{C}, \lambda>-p$ and $\Re(\alpha) \geq 0$. Then

(1) The inclusion $R_{a, c}^{\lambda}(p, \alpha, \mu) \subset R_{a, c}^{\lambda}\left(p, \alpha_{1}, \mu_{1}\right)$ holds whenever $, \alpha_{1} \in \mathbb{C}, \Re\left(\alpha_{1}\right) \geq$ 0 , and $\mu_{1}$ is defined by $\mu_{1}=\frac{\mu}{|\alpha|}\left(\frac{p\left|\alpha-\alpha_{1}\right|}{|\alpha+p|}+\left|\alpha_{1}\right|\right)$.

(2) The inclusion $R_{a, c}^{\lambda}(p, \alpha, \mu) \subset R_{a+1, c}^{\lambda}\left(p, \alpha, \mu_{1}\right)$ holds whenever $\mu_{1}=\mu \frac{a}{a+1}$.

(3) If $f_{1} \in R_{a, c}^{\lambda}\left(p, \alpha_{1}, \mu_{1}\right)$ and $f_{2} \in R_{a, c}^{\lambda}\left(p, \alpha_{2}, \mu_{2}\right)$, then $F(z)=\tau_{p}^{\lambda}(a+1, c)\left(f_{1} *\right.$ $\left.f_{2}\right)(z) \in R_{a, c}^{\lambda}\left(p, \alpha_{1}, \mu_{1}\right)$, whenever $0 \neq \alpha_{j} \in \mathbb{C}, \Re\left(\alpha_{j}\right) \geq 0,(j=1,2)$, and $0<\mu_{2} \leq \frac{\left|\alpha_{2}+p\right|(a+1)}{a p}$.

Proof. We first prove (1). Let

$$
p(z)=\frac{\tau_{p}^{\lambda}(a, c) f(z)}{z^{p}}
$$

for $f \in R_{a, c}^{\lambda}(p, \alpha, \mu)$, then $p(z)$ is analytic in $\mathbb{U}$ and

$$
\tau_{p}^{\lambda}(a, c) f(z)=z^{p} p(z) .
$$

By taking the derivative in the both sides of the above equation, we obtain

$$
(1-\alpha) \frac{\tau_{p}^{\lambda}(a, c) f(z)}{z^{p}}+\frac{\alpha}{p} \frac{z\left(\tau_{p}^{\lambda}(a, c) f(z)\right)^{\prime}}{z^{p}}=p(z)+\frac{\alpha}{p} z p^{\prime}(z) .
$$

Since $f \in R_{a, c}^{\lambda}(p, \alpha, \mu)$, Lemma 1.1 and (2.1) implies that

$$
\frac{\tau_{p}^{\lambda}(a, c) f(z)}{z^{p}} \prec 1+\frac{\mu p}{\alpha+p} z .
$$

We now consider the identity

$$
\begin{aligned}
& \alpha\left[\left(1-\alpha_{1}\right) \frac{\tau_{p}^{\lambda}(a, c) f(z)}{z^{p}}+\frac{\alpha_{1}}{p} \frac{z\left(\tau_{p}^{\lambda}(a, c) f(z)\right)^{\prime}}{z^{p}}-1\right] \\
= & \left(\alpha-\alpha_{1}\right)\left(\frac{\tau_{p}^{\lambda}(a, c) f(z)}{z^{p}}-1\right) \\
& +\alpha_{1}\left[(1-\alpha) \frac{\tau_{p}^{\lambda}(a, c) f(z)}{z^{p}}+\frac{\alpha}{p} \frac{z\left(\tau_{p}^{\lambda}(a, c) f(z)\right)^{\prime}}{z^{p}}-1\right],
\end{aligned}
$$


which holds for all $\alpha$ and $\alpha_{1}$. It follows that

$$
\left|\left(1-\alpha_{1}\right) \frac{\tau_{p}^{\lambda}(a, c) f(z)}{z^{p}}+\frac{\alpha_{1}}{p} \frac{z\left(\tau_{p}^{\lambda}(a, c) f(z)\right)^{\prime}}{z^{p}}-1\right| \leq \frac{\mu}{|\alpha|}\left(\frac{p\left|\alpha-\alpha_{1}\right|}{|\alpha+p|}+\left|\alpha_{1}\right|\right),
$$

and the desired inclusion relationship is clear.

(2) Let $f \in R_{a, c}^{\lambda}(p, \alpha, \mu)$ and

$$
q(z)=(1-\alpha) \frac{\tau_{p}^{\lambda}(a+1, c) f(z)}{z^{p}}+\frac{\alpha}{p} \frac{z\left(\tau_{p}^{\lambda}(a+1, c) f(z)\right)^{\prime}}{z^{p}} .
$$

Using (1.6) and by taking derivative of (1.6), we obtain

$$
q(z)+\frac{z q^{\prime}(z)}{a}=(1-\alpha) \frac{\tau_{p}^{\lambda}(a, c) f(z)}{z^{p}}+\frac{\alpha}{p} \frac{z\left(\tau_{p}^{\lambda}(a, c) f(z)\right)^{\prime}}{z^{p}} .
$$

By applying Lemma 1.1 to (2.2), we can write

$$
q(z) \prec 1+\mu \frac{a}{a+1} z .
$$

Thus

$$
f \in R_{a, c}^{\lambda}\left(p, \alpha, \mu \frac{a}{a+1}\right) .
$$

The estimate $\mu \frac{a}{a+1}$ cannot be improved in general.

(3) Let $f_{j} \in R_{a, c}^{\lambda}\left(p, \alpha_{j}, \mu_{j}\right)(j=1,2)$ and $\phi(z)=\frac{\tau_{p}^{\lambda}(a, c) F(z)}{z^{p}}$. Noting that

$$
\begin{aligned}
& \left(1-\alpha_{1}\right) \frac{\tau_{p}^{\lambda}(a, c) F(z)}{z^{p}}+\frac{\alpha_{1}}{p} \frac{z\left(\tau_{p}^{\lambda}(a, c) F(z)\right)^{\prime}}{z^{p}} \\
= & \phi(z)+\frac{\alpha_{1}}{p} z \phi^{\prime}(z) \\
= & \phi(z) * \phi_{\alpha_{1}, p}(z) \\
= & \frac{\tau_{p}^{\lambda}(a, c) f_{1}(z)}{z^{p}} * \frac{\tau_{p}^{\lambda}(a+1, c) f_{2}(z)}{z^{p}} * \phi_{\alpha_{1}, p}(z) \\
= & \frac{\tau_{p}^{\lambda}(a, c) f_{1}(z)}{z^{p}} * \phi_{\alpha_{1}, p}(z) * \frac{\tau_{p}^{\lambda}(a+1, c) f_{2}(z)}{z^{p}},
\end{aligned}
$$

where

$$
\phi_{\alpha_{1}, p}(z)=1+\sum_{k=1}^{\infty}\left(1+\frac{\alpha_{1}}{p} k\right) z^{k}
$$


By assumption and using Lemma 1.1, we observe that $(2.3)$

$\frac{\tau_{p}^{\lambda}(a, c) f_{1}(z)}{z^{p}} * \phi_{\alpha_{1}, p}(z) \prec 1+\mu_{1} z \quad$ and $\quad \frac{\tau_{p}^{\lambda}(a+1, c) f_{2}(z)}{z^{p}} \prec 1+\frac{a \mu_{2} p}{(a+1)\left(\alpha_{2}+p\right)} z$.

Since the functions $1+\mu_{1} z$ and $1+\frac{a \mu_{2} p}{(a+1)\left(\alpha_{2}+p\right)} z$ are convex in $\mathbb{U}$, by applying Lemma 1.2 to (2.3), we conclude the desired result.

Upon setting $a=p=\lambda=c=1$ in the third part of Theorem 2.1, we arrive at the following result.

Corollary 2.1. Let $0 \neq \alpha_{j} \in \mathbb{C}, \Re\left(\alpha_{j}\right) \geq 0(j=1,2)$ and the functions $f_{j}(z) \in$ $\mathcal{A}(j=1,2)$ satisfy the following subordination:

$$
f_{j}^{\prime}(z)+\alpha_{j} z f_{j}^{\prime \prime}(z) \prec 1+\mu_{j} z \quad(j=1,2)
$$

then

$$
\left(f_{1} * f_{2}\right)^{\prime}(z)+\alpha_{1} z\left(f_{1} * f_{2}\right)^{\prime \prime}(z) \prec 1+\mu_{1} z,
$$

whenever $\mu_{2}<2\left|\alpha_{2}+1\right|$.

Theorem 2.2. Let $a>0,0 \neq \alpha \in \mathbb{C}, \Re(\alpha) \geq 0, f \in R_{a, c}^{\lambda}(p, \alpha, \mu)$ and

$$
0<\mu \leq \frac{(a+1)|\alpha+p| \sin \frac{\pi}{2} \delta}{p \sqrt{1+2 a(a+1)\left(1+\cos \frac{\pi}{2} \delta\right)}}
$$

Then

$$
\left|\arg \frac{\tau_{p}^{\lambda}(a, c) f(z)}{\tau_{p}^{\lambda}(a+1, c) f(z)}\right|<\frac{\pi}{2} \delta, \quad(0<\delta \leq 1) .
$$

Proof. By Lemma 1.1 and Theorem 2.1, we obtain

$$
\left|\arg \frac{\tau_{p}^{\lambda}(a, c) f(z)}{z^{p}}\right|<\arcsin \frac{\mu p}{|\alpha+p|}
$$

and

$$
\left|\arg \frac{\tau_{p}^{\lambda}(a+1, c) f(z)}{z^{p}}\right|<\arcsin \frac{\mu a p}{(a+1)|\alpha+p|} .
$$


Thus, we have

$$
\begin{aligned}
\left|\arg \frac{\tau_{p}^{\lambda}(a, c) f(z)}{\tau_{p}^{\lambda}(a+1, c) f(z)}\right| \leq & \left|\arg \frac{\tau_{p}^{\lambda}(a+1, c) f(z)}{z^{p}}\right|+\left|\arg \frac{\tau_{p}^{\lambda}(a, c) f(z)}{z^{p}}\right| \\
\leq & \arcsin \frac{(a+1) \sin \frac{\pi}{2} \delta}{\sqrt{1+2 a(a+1)\left(1+\cos \frac{\pi}{2} \delta\right)}} \\
& +\arcsin \frac{a \sin \frac{\pi}{2} \delta}{\sqrt{1+2 a(a+1)\left(1+\cos \frac{\pi}{2} \delta\right)}} \\
= & \frac{\pi}{2} \delta .
\end{aligned}
$$

This completes the proof of Theorem 2.2.

Upon setting $a=p=\lambda=c=1$ in Theorem 2.2, and using Corollary 2.1, we get the following result.

Corollary 2.2. Let $0 \neq \alpha_{j} \in \mathbb{C}, \Re\left(\alpha_{j}\right) \geq 0(j=1,2)$ and the functions $f_{j}(z) \in$ $\mathcal{A}(j=1,2)$ satisfy the following subordination:

$$
f_{j}^{\prime}(z)+\alpha_{j} z f_{j}^{\prime \prime}(z) \prec 1+\mu_{j} z \quad(j=1,2),
$$

then

$$
f_{1} * f_{2} \in S(\delta)
$$

where $0<\delta \leq 1$ and other parameters are related to the inequality

$$
0<\frac{\mu_{1} \mu_{2}}{\left|\left(1+\alpha_{1}\right)\left(1+\alpha_{2}\right)\right|} \leq \frac{4 \sin \left(\frac{\pi \delta}{2}\right)}{\sqrt{5+4 \cos \left(\frac{\pi \delta}{2}\right)}}
$$

For a function $f(z) \in \mathcal{A}_{p}$, the integral operator $F_{\mu, p}: \mathcal{A}_{p} \mapsto \mathcal{A}_{p}$ is defined by

$$
\begin{aligned}
& F_{\mu, p}(f)(z)=\frac{\mu+p}{z^{\mu}} \int_{0}^{z} t^{\mu-1} f(t) d t \\
& =\left(z^{p}+\sum_{k=1}^{\infty} \frac{\mu+p}{\mu+p+k} z^{p+k}\right) * f(z) \quad(\mu>-p ; z \in \mathbb{U}) .
\end{aligned}
$$

Theorem 2.3. If $f \in R_{a, c}^{\lambda}(p, \alpha, M)$, then the function $F_{\mu, p}(f)$ defined by (2.4) belongs to the class $R_{a, c}^{\lambda}\left(p, \alpha, M_{1}\right)$, where $M_{1}=M \frac{\mu+p}{\mu+p+1}$.

Proof. It follows from (1.6) and (2.5) that

$$
z\left(\tau_{p}^{\lambda}(a, c) F_{\mu, p}(f)(z)\right)^{\prime}=(\mu+p) \tau_{p}^{\lambda}(a, c) f(z)-\mu \tau_{p}^{\lambda}(a, c) F_{\mu, p}(f)(z) .
$$


We now put

$$
\varphi(z)=(1-\alpha) \frac{\tau_{p}^{\lambda}(a, c) F_{\mu, p}(f)(z)}{z^{p}}+\frac{\alpha}{p} \frac{z\left(\tau_{p}^{\lambda}(a, c) F_{\mu, p}(f)(z)\right)^{\prime}}{z^{p}} .
$$

Using (2.6) and the differentiation of (2.4), we find that

$$
(1-\alpha) \frac{\tau_{p}^{\lambda}(a, c) f(z)}{z^{p}}+\frac{\alpha}{p} \frac{z\left(\tau_{p}^{\lambda}(a, c) f(z)\right)^{\prime}}{z^{p}}=\varphi(z)+\frac{z \varphi^{\prime}(z)}{\mu+p} .
$$

Since $f \in R_{a, c}^{\lambda}(p, \alpha, M)$, by virtue of (2.8), we have

$$
\varphi(z)+\frac{z \varphi^{\prime}(z)}{\mu+p} \prec 1+M z .
$$

Thus, by Lemma 1.1, we get

$$
\varphi(z) \prec 1+M \frac{\mu+p}{\mu+p+1} z .
$$

We thus complete the proof of Theorem 2.3.

By putting $a=p=\lambda=c=1$ in Theorems 2.2 and 2.3, we deduce the following consequence.

Corollary 2.3. Let $0 \neq \alpha \in \mathbb{C}, \Re(\alpha) \geq 0$ and the function $f(z) \in \mathcal{A}$ satisfy the following subordination

$$
f^{\prime}(z)+\alpha z f^{\prime \prime}(z) \prec 1+M z
$$

then

$$
F_{\mu, 1}(f)(z) \in \mathcal{S}(\delta)
$$

where $M<\frac{\mu+2}{\mu+1} \frac{2 \sin \left(\frac{\pi \delta}{2}\right)}{\sqrt{5+4 \cos \left(\frac{\pi \delta}{2}\right)}}$.

Theorem 2.4. Let $0 \neq \alpha \in \mathbb{C}, \Re\left(\frac{1}{\alpha}\right)>-\frac{1}{p}, 0<\mu p<|\alpha|\left(1+\Re\left(\frac{p}{\alpha}\right)\right), 0<m|a| \leq$ $1+\Re(a)$ and $f \in R_{a, c}^{\lambda}(p, \alpha, \mu)$. Then

$$
\left|\frac{z\left(\tau_{p}^{\lambda}(a, c) f(z)\right)^{\prime}}{\tau_{p}^{\lambda}(a, c) f(z)}-p\right|<\frac{\mu p\left[|\alpha|\left(1+\Re\left(\frac{p}{\alpha}\right)\right)+p\right]}{|\alpha|\left[|\alpha|\left(1+\Re\left(\frac{p}{\alpha}\right)\right)-\mu p\right]},
$$

and

$$
\left|\frac{z\left(\tau_{p}^{\lambda}(a+1, c) f(z)\right)^{\prime}}{\tau_{p}^{\lambda}(a+1, c) f(z)}-p\right|<\frac{m|a|[1+\Re(a)+|a|]}{1+\Re(a)-m|a|},
$$

where

$$
m=\frac{\mu p}{|\alpha|\left(1+\Re\left(\frac{p}{\alpha}\right)\right)} .
$$


Proof. Assume that $f \in \mathcal{A}_{p}$ satisfies the condition (1.10). By setting

$$
p(z)=\frac{\tau_{p}^{\lambda}(a, c) f(z)}{z^{p}},
$$

and by (1.10), we have

$$
p(z)+\frac{\alpha}{p} z p^{\prime}(z)=1+\mu \omega(z),
$$

where $\omega \in B$. After some algebraic calculations, from (2.8), we obtain

$$
p(z)=1+\mu \frac{p}{\alpha} \int_{0}^{1} t^{\frac{p}{\alpha}-1} \omega(t z) d t=1+\mu \frac{p}{\alpha} \sum_{n=1}^{\infty} \frac{1}{n+\frac{p}{\alpha}} \omega_{n} z^{n} .
$$

where

$$
\omega(z)=\sum_{n=1}^{\infty} \omega_{n} z^{n} .
$$

It follows that

$$
\begin{aligned}
& (z p(z))^{\prime}=1+\mu \frac{p}{\alpha} \sum_{n=1}^{\infty} \frac{n+1}{n+\frac{p}{\alpha}} \omega_{n} z^{n} \\
& =1+\mu \frac{p}{\alpha} \sum_{n=1}^{\infty} \frac{1}{n+\frac{p}{\alpha}} \omega_{n} z^{n}+\mu \frac{p}{\alpha}\left(\omega(z)-\frac{p}{\alpha} \int_{0}^{1} t^{\frac{p}{\alpha}-1} \omega(t z) d t\right) .
\end{aligned}
$$

In particular,

$$
z p^{\prime}(z)=\mu \frac{p}{\alpha}\left(\omega(z)-\frac{p}{\alpha} \int_{0}^{1} t^{\frac{p}{\alpha}-1} \omega(t z) d t\right)
$$

and therefore, from (2.9) and (2.10), we get

$$
\left|\frac{z p^{\prime}(z)}{p(z)}\right|<\frac{\mu p\left[|\alpha|\left(1+\Re\left(\frac{p}{\alpha}\right)\right)+p\right]}{|\alpha|\left[|\alpha|\left(1+\Re\left(\frac{p}{\alpha}\right)\right)-\mu p\right]},
$$

or equivalently

$$
\left|\frac{z\left(\tau_{p}^{\lambda}(a, c) f(z)\right)^{\prime}}{\tau_{p}^{\lambda}(a, c) f(z)}-p\right|<\frac{\mu p\left[|\alpha|\left(1+\Re\left(\frac{p}{\alpha}\right)\right)+p\right]}{|\alpha|\left[|\alpha|\left(1+\Re\left(\frac{p}{\alpha}\right)\right)-\mu p\right]} .
$$

For proving the second inequality, by setting

$$
m=\frac{\mu p}{|\alpha|\left(1+\Re\left(\frac{p}{\alpha}\right)\right)}, \quad p_{1}(z)=\frac{\tau_{p}^{\lambda}(a+1, c) f(z)}{z^{p}}
$$


and making use of (2.10) and (1.6), we can write

$$
z p_{1}^{\prime}(z)+a p_{1}(z)=a+m a \omega_{1}(z)
$$

where $\omega_{1}(z) \in B$. After some similar computations as above, it follows that

$$
p_{1}(z)=1+m a \int_{0}^{1} t^{a-1} \omega_{1}(t z) d t
$$

and

$$
z p_{1}^{\prime}(z)=m a\left(\omega_{1}(z)-a \int_{0}^{1} t^{a-1} \omega_{1}(t z) d t\right)
$$

Hence

$$
\left|p_{1}(z)\right| \geq 1-m|a| \frac{1}{1+\Re(a)}, \quad\left|z p_{1}^{\prime}(z)\right| \leq m|a|\left(1+\frac{|a|}{1+\Re(a)}\right),
$$

and the desired conclusion follows from the above inequalities.

Upon setting $a=p=\lambda=c=1$ in Theorems 2.4, we get the following consequence.

Corollary 2.4. Let $0<\alpha<1,0<\mu<1$ and function $f(z) \in \mathcal{A}$ satisfy the following subordination

$$
f^{\prime}(z)+\alpha z f^{\prime \prime}(z) \prec 1+\mu z,
$$

then

$$
\left|\frac{z f^{\prime \prime}(z)}{f^{\prime}(z)}\right|<\frac{\mu[\alpha+2]}{\alpha[\alpha+1-\mu]}
$$

and

$$
\left|\frac{z f^{\prime}(z)}{f(z)}-1\right|<\frac{3 \mu}{2(\alpha+1)-\mu}
$$

Theorem 2.5. Let $0 \neq \alpha \in \mathbb{C}, \Re\left(\frac{1}{\alpha}\right)>-\frac{1}{p}$ and $0 \leq \gamma<1$. If $f \in R_{a, c}^{\lambda}(p, \alpha, \mu)$, then $\tau_{p}^{\lambda}(a, c) f(z) \in \mathcal{S}^{*}(\gamma)$ whenever $\mu$ satisfies the inequality

$$
0 \leq \mu<\frac{(p-\gamma)\left(|\alpha|^{2}+p \Re(\alpha)\right)}{p\left[|\alpha|+\frac{p \Re(\alpha)}{|\alpha|}+1+(p-\gamma)|\alpha|\right]} .
$$

Proof. Let

$$
p(z)=\frac{\tau_{p}^{\lambda}(a, c) f(z)}{z^{p}}
$$


in view of the representations (2.9) and (2.10), we have

$$
\frac{1}{p-\gamma}\left(\frac{z p^{\prime}(z)}{p(z)}+p-\gamma\right)=\frac{1}{p-\gamma}\left[\frac{\mu \frac{p}{\alpha}\left(\omega(z)-\frac{p}{\alpha} \int_{0}^{1} t^{\frac{p}{\alpha}-1} \omega(t z) d t\right)}{1+\mu \frac{p}{\alpha} \int_{0}^{1} t^{\frac{p}{\alpha}-1} \omega(t z) d t}+p-\gamma\right]
$$

where $\omega \in B$. We need to show that $\tau_{p}^{\lambda}(a, c) f(z) \in \mathcal{S}^{*}(\gamma)$. But according to a well known result obtained in [6], and in view of equality

$$
\frac{z p^{\prime}(z)}{p(z)}=\frac{z\left(\tau_{p}^{\lambda}(a, c) f(z)\right)^{\prime}}{\tau_{p}^{\lambda}(a, c) f(z)}+p
$$

it suffices to show that

$$
\frac{1}{p-\gamma}\left(\frac{z p^{\prime}(z)}{p(z)}+p-\gamma\right) \neq-i T, \quad T \in \mathbb{R}
$$

However, by (2.13), it can be easily seen that this is equivalent to verify that

$$
\mu\left\{\frac{p}{\alpha(p-\gamma)(1+i T)}\left[\omega(z)-\frac{p}{\alpha} \int_{0}^{1} t^{\frac{p}{\alpha}-1} \omega(t z) d t\right]+\frac{p}{\alpha} \int_{0}^{1} t^{\frac{p}{\alpha}-1} \omega(t z) d t\right\} \neq-1 .
$$

Now, if we let

$$
M=\sup _{T \in \mathbb{R}, \omega \in B}\left|\frac{p}{\alpha(p-\gamma)(1+i T)}\left[\omega(z)-\frac{p}{\alpha} \int_{0}^{1} t^{\frac{p}{\alpha}-1} \omega(t z) d t\right]+\frac{p}{\alpha} \int_{0}^{1} t^{\frac{p}{\alpha}-1} \omega(t z) d t\right| .
$$

Then, in view of the rotation invariance of $B, \tau_{p}^{\lambda}(a, c) f(z) \in \mathcal{S}^{*}(\gamma)$, if $\mu M \leq 1$. Now, for $\Re\left(\frac{1}{\alpha}\right)>-R\left(\frac{1}{p}\right)$, we observe that

$$
\begin{aligned}
M & \leq \frac{p}{|\alpha|(p-\gamma)}\left|\omega(z)-\frac{p}{\alpha} \int_{0}^{1} t^{\frac{p}{\alpha}-1} \omega(t z) d t\right|+\frac{p}{|\alpha|}\left|\int_{0}^{1} t^{\frac{p}{\alpha}-1} \omega(t z) d t\right| \\
& \leq \frac{p}{|\alpha|(p-\gamma)}\left(1+\frac{p}{|\alpha|\left(1+\Re\left(\frac{p}{\alpha}\right)\right)}\right)+\frac{p}{|\alpha|\left(1+\Re\left(\frac{p}{\alpha}\right)\right)} \\
& =\frac{p\left(|\alpha|\left(1+\Re\left(\frac{p}{\alpha}\right)\right)+1+(p-\gamma)|\alpha|\right)}{|\alpha|^{2}(p-\gamma)\left(1+\Re\left(\frac{p}{\alpha}\right)\right)}
\end{aligned}
$$

which shows that

$$
M=\frac{p\left(|\alpha|\left(1+\Re\left(\frac{p}{\alpha}\right)\right)+1+(p-\gamma)|\alpha|\right)}{|\alpha|^{2}(p-\gamma)\left(1+\Re\left(\frac{p}{\alpha}\right)\right)} .
$$

Therefore, $\tau_{p}^{\lambda}(a, c) f(z) \in \mathcal{S}^{*}(\gamma)$, whenever $\mu M \leq 1$. The desired conclusion follows from the hypotheses. 
Putting $a=2, \lambda=1, c=1, p=1$ and $a=1, \lambda=1, c=1, p=1$ in Theorem 2.5 , we get the following results.

Corollary 2.5. Let $0 \neq \alpha \in \mathbb{C}, \Re\left(\frac{1}{\alpha}\right)>-1$ and $0 \leq \gamma<1$. If $f \in \mathcal{A}$ satisfies the condition:

$$
(1-\alpha) \frac{f(z)}{z}+\alpha f^{\prime}(z) \prec 1+\mu z
$$

where

$$
0 \leq \mu<\frac{(1-\gamma)\left(|\alpha|^{2}+\Re(\alpha)\right)}{|\alpha|+\frac{\Re(\alpha)}{|\alpha|}+1+(1-\gamma)|\alpha|}
$$

then $f \in \mathcal{S}^{*}(\gamma)$.

Corollary 2.6. Let $0 \neq \alpha \in \mathbb{C}, \Re\left(\frac{1}{\alpha}\right)>-1$ and $0 \leq \gamma<1$. If $f \in \mathcal{A}$ satisfies the condition:

$$
f^{\prime}(z)+\alpha z f^{\prime \prime}(z) \prec 1+\mu z,
$$

and $\mu$ satisfy $(2.14)$, then $z f^{\prime} \in \mathcal{S}^{*}(\gamma)$.

Corollary 2.7. Let $0 \neq \alpha \in \mathbb{C}, \Re\left(\frac{1}{\alpha}\right)>-1$ and $0 \leq \gamma<1$. If $f \in \mathcal{A}$ satisfies the condition:

$$
(1-\alpha) \frac{f(z)}{z}+\alpha f^{\prime}(z) \prec 1+M z
$$

where

$$
0 \leq M<\frac{(2+\mu)(1-\gamma)\left(|\alpha|^{2}+\Re(\alpha)\right)}{(1+\mu)\left(|\alpha|+\frac{\Re(\alpha)}{|\alpha|}+1+(1-\gamma)|\alpha|\right)}
$$

then $F_{\mu, 1}(f) \in \mathcal{S}^{*}(\gamma)$.

Theorem 2.6. Let $\alpha>0, a>0,0 \leq \gamma \leq \frac{1}{2}$ and $f \in R_{a, c}^{\lambda}(p, \alpha, \mu)$. If $\mu$ satisfies the following conditions:

$0<\mu \leq \begin{cases}\frac{4(1-\gamma)\left(\frac{p}{\alpha}+1\right)(a+1) \alpha}{p\left[\left(\frac{p}{\alpha}+1\right)(1+a)+2 a \alpha(1-2 \gamma)+2+4 a\left(\frac{a \alpha}{p}\right)^{\frac{a \alpha}{p-a \alpha}}\right]}, & \left(0<\alpha<\infty ; \alpha \neq \frac{p}{a}\right) ; \\ \frac{4(1-\gamma)(a+1)^{2}}{a\left[(1+a)^{2}+2 p(1-2 \gamma)+2+4 a e^{\left.\frac{-(a+1)}{a}\right]},\right.} & \left(\alpha=\frac{p}{a}\right) .\end{cases}$

then

$$
\Re\left(\frac{\tau_{p}^{\lambda}(a, c) f(z)}{\tau_{p}^{\lambda}(a+1, c) f(z)}\right)>\gamma
$$


Proof. Let $p(z)=\frac{\tau_{p}^{\lambda}(a, c) f(z)}{z^{p}}$ and $p_{1}(z)=\frac{\tau_{p}^{\lambda}(a+1, c) f(z)}{z^{p}}$. In view of the representation (2.10), we have

$$
p(z)=1+\mu \frac{p}{\alpha} \int_{0}^{1} t^{\frac{p}{\alpha}-1} \omega(t z) d t .
$$

Using the identity (2.17) in (1.6) and carrying out Lemma 1.2 in the resulting equation, we deduce that

$$
p_{1}(z)= \begin{cases}1+a \frac{\mu p}{p-a \alpha} \int_{0}^{1}\left(t^{a-1}-t^{\frac{p}{\alpha}-1}\right) \omega(t z) d t, & \left(\alpha \neq \frac{p}{a}\right) ; \\ 1+a \frac{\mu p}{\alpha} \int_{0}^{1} t^{a-1} \log \frac{1}{t} \omega(t z) d t, & \left(\alpha=\frac{p}{a}\right) .\end{cases}
$$

We need to consider the two cases $\alpha=\frac{p}{a}$ and $\alpha \neq \frac{p}{a}$. Using the last two equations, we see that for $\alpha \neq \frac{p}{a}$,

$$
\frac{1}{1-\gamma}\left(\frac{p(z)}{p_{1}(z)}-\gamma\right)=\frac{1}{1-\gamma}\left(\frac{1+\mu \frac{p}{\alpha} \int_{0}^{1} t^{\frac{p}{\alpha}-1} \omega(t z) d t}{1+a \frac{\mu p}{p-a \alpha} \int_{0}^{1}\left(t^{a-1}-t^{\frac{p}{\alpha}-1}\right) \omega(t z) d t}-\gamma\right)
$$

For proving (2.16), it suffices to show that

$$
\frac{1}{1-\gamma}\left(\frac{p(z)}{p_{1}(z)}-\gamma\right) \neq i T, \quad T \in \mathbb{R} .
$$

But, by (2.18), it can be easily seen that this is equivalent to verify that

$$
\begin{gathered}
\frac{\mu}{2}\left[\int_{0}^{1}\left(\frac{p}{\alpha(1-\gamma)} t^{\frac{p}{\alpha}-1}-a \frac{p(2 \gamma-1)}{(p-a \alpha)(1-\gamma)}\left(t^{a-1}-t^{\frac{p}{\alpha}-1}\right)\right) \omega(t z) d t\right]+ \\
\frac{\mu}{2}\left[\frac{1+i T}{1-i T} \int_{0}^{1}\left(\frac{p}{\alpha(1-\gamma)} t^{\frac{p}{\alpha}-1}-a \frac{p}{(p-a \alpha)(1-\gamma)}\left(t^{a-1}-t^{\frac{p}{\alpha}-1}\right)\right) \omega(t z) d t\right] \neq-1,
\end{gathered}
$$

which is same as

$$
\begin{gathered}
\frac{\mu}{2} \frac{p}{\alpha(p-a \alpha)(1-\gamma)}\left[\int_{0}^{1}\left[(p-a \alpha+a \alpha(2 \gamma-1)) t^{\frac{p}{\alpha}-1}-a \alpha(2 \gamma-1) t^{a-1}\right] \omega(t z) d t\right] \\
+\frac{\mu}{2} \frac{p}{\alpha(p-a \alpha)(1-\gamma)}\left[\frac{1+i T}{1-i T} \int_{0}^{1}\left(p t^{\frac{p}{\alpha}-1}-a \alpha t^{a-1}\right) \omega(t z) d t\right] \neq-1 .
\end{gathered}
$$

Now, if we let

$$
M=\sup _{T \in \mathbb{R}, \omega \in B}\left|\frac{p}{\alpha(p-a \alpha)(1-\gamma)}\right| \times
$$




$$
\begin{gathered}
\mid \int_{0}^{1}\left[(p-a \alpha+a \alpha(2 \gamma-1)) t^{\frac{p}{\alpha}-1}-a \alpha(2 \gamma-1) t^{a-1}\right] \omega(t z) d t \\
+\frac{1+i T}{1-i T} \int_{0}^{1}\left(p t^{\frac{p}{\alpha}-1}-a \alpha t^{a-1}\right) \omega(t z) d t \mid
\end{gathered}
$$

Then, in view of the rotation property of the class $B$, we obtain that

$$
\Re\left(\frac{p(z)}{p_{1}(z)}\right)>\gamma \quad \text { if } \quad \mu M \leq 2 .
$$

Thus our aim is to find the value of $M$. We consider the positiveness of the integrand in (2.19). It is easy to see that

$$
\frac{(p-a \alpha+a \alpha(2 \gamma-1)) t^{\frac{p}{\alpha}-1}-a \alpha(2 \gamma-1) t^{a-1}}{p-a \alpha}
$$

is positive for all $t \in[01], \alpha \neq \frac{p}{a}$ and $0 \leq \gamma \leq \frac{1}{2}$, also

$$
\frac{p t^{\frac{p}{\alpha}-1}-a \alpha t^{a-1}}{p-a \alpha}
$$

is positive for $t>\left(\frac{a \alpha}{p}\right)^{\frac{\alpha}{p-a \alpha}}$ and is negative for $0 \leq t \leq\left(\frac{a \alpha}{p}\right)^{\frac{\alpha}{p-a \alpha}}$. Hence, for $\alpha \neq \frac{p}{a}$, using the above observations, we estimate that

$$
\begin{aligned}
& \quad M<\frac{p}{\alpha(p-a \alpha)(1-\gamma)} \int_{0}^{1}\left[(p-a \alpha+a \alpha(2 \gamma-1)) t^{\frac{p}{\alpha}-1}-a \alpha(2 \gamma-1) t^{a-1}\right] t d t \\
& +\frac{p}{\alpha(p-a \alpha)(1-\gamma)}\left[\int_{0}^{t_{1}}\left(-p t^{\frac{p}{\alpha}-1}+a \alpha t^{a-1}\right) t d t+\int_{t_{1}}^{1}\left(p t^{\frac{p}{\alpha}-1}-a \alpha t^{a-1}\right) t d t\right] \\
& =\frac{p}{2(1-\gamma)\left(\frac{p}{\alpha}+1\right)(1+a) \alpha}\left[\left(\frac{p}{\alpha}+1\right)(1+a)-2 a \alpha(2 \gamma-1)+2+4 a\left(\frac{a \alpha}{p}\right)^{\frac{a \alpha}{p-a \alpha}}\right], \\
& \text { where } t_{1}=\left(\frac{a \alpha}{p}\right)^{\frac{\alpha}{p-a \alpha}} .
\end{aligned}
$$

Therefore, we find that

$$
M=\frac{p\left[\left(\frac{p}{\alpha}+1\right)(1+a)-2 a \alpha(2 \gamma-1)+2+4 a\left(\frac{a \alpha}{p}\right)^{\frac{a \alpha}{p-a \alpha}}\right]}{2(1-\gamma)\left(\frac{p}{\alpha}+1\right)(1+a) \alpha} .
$$


Next, we deal the case $\alpha=\frac{p}{a}$. We observe that

$$
\lim _{\alpha \mapsto \frac{p}{a}} \frac{p\left[(p-a \alpha)-a \alpha(2 \gamma-1) t^{a-1}\left(1-t^{\frac{p}{\alpha}-a}\right)\right]}{\alpha(p-a \alpha)(1-\gamma)}=\frac{p\left[1+a(1-2 \gamma) t^{a-1} \log \frac{1}{t}\right]}{\alpha(1-\gamma)}>0 .
$$

Similarly, as $\alpha \mapsto \frac{p}{\alpha}$, one has

$\frac{p\left(p t^{\frac{p}{\alpha}-1}-a \alpha t^{a-1}\right)}{\alpha(p-a \alpha)(1-\gamma)}=\frac{p t^{a-1}}{\alpha(1-\gamma)}\left(t^{\frac{p}{\alpha}-a}+a \frac{t^{\frac{p}{\alpha}-a}-1}{\frac{p}{\alpha}-a}\right) \mapsto \frac{p t^{a-1}}{\alpha(1-\gamma)}(1+a \log t)>0$,

for all $t \in\left[e^{-a} 1\right]$. Therefore for $\alpha=\frac{p}{a}$, we can easily obtain

$$
M=\frac{p\left[(a+1)^{2}+2 p(1-2 \gamma)+2+4 a e^{-\left(\frac{a+1}{a}\right)}\right]}{2(1-\gamma)(a+1)^{2} \alpha} .
$$

The proof of Theorem 2.6 is thus completed.

By putting $a=p=\lambda=c=1$ in Theorem 2.6, we get the following result.

Corollary 2.8. Let $\alpha>0$ and $0 \leq \gamma \leq \frac{1}{2}$. If $f \in \mathcal{A}$ satisfies the following condition:

$$
f^{\prime}(z)+\alpha z f^{\prime \prime}(z) \prec 1+\mu z,
$$

where

$$
0<\mu \leq \begin{cases}\frac{4(1-\gamma)\left(\frac{1}{\alpha}+1\right) \alpha}{\left(\frac{1}{\alpha}+1\right)+\alpha(1-2 \gamma)+1+2 \alpha^{\frac{\alpha}{1-\alpha}}}, & (0<\alpha<\infty ; \alpha \neq 1) ; \\ \frac{4(1-\gamma)^{2}}{2+(1-2 \gamma)+1+2 e^{-2}}, & (\alpha=1),\end{cases}
$$

then $f \in \mathcal{S}^{*}(\gamma)$.

Acknowledgment. The present investigation was supported, in part, by the $H u$ nan Provincial Natural Science Foundation under Grant 05JJ30013 and the Scientific Research Fund of Hunan Provincial Education Department under Grant 05C266 of People's Republic of China. I would like to thank the referee for their careful reading and making valuable comments which have essentially improved the presentation of this paper.

\section{References}

[1] R. Aghalary, On subclasses of p-valent analytic functions defined by integral operators, Kyungpook Math. J., 47(2007), 393-401. 
[2] N. E. Cho, O. S. Kwon and H. M. Srivastava, Inclusion relationships and argument properties for certain subclasses of multivalent functions associated with a family of linear operators, J. Math. Anal. Appl., 292(2004), 470-483.

[3] D. J. Hallenbeck and St. Ruscheweyh, Subordination by convex functions, Proc. Amer. Math. Soc., 52(1975), 191-195.

[4] J. Patel, On certain subclasses of multivalent functions involving Cho-KwonSrivastava operator, Ann. Univ. Mariae Curie-Skłodowska Sect. A, 60(2006), 75-86.

[5] St. Ruscheweyh and J. Stankiewicz, Subordination and convex univalent functions, Bull. Pol. Acad. Sci. Math., 33(1985), 499-502.

[6] S. Ruscheweyh, New ceriteria for univalent functions, Proc. Amer. Math. Soc., 49(1975), 109-115.

[7] J. Sokół and L. Trojnar-Spelina, Convolution properties for certain classes of multivalent functions, J. Math. Anal. Appl., 337(2008), 1190-1197. 